\title{
Consideraciones sobre el concepto de destrucción fenomenológica en Martin Heidegger. A cien años de las lecciones de 1919-1920*
}

\author{
Carlos Arturo Bedoya Rodas \\ Facultad de Filosofía, Universidad Pontificia Bolivariana, Medellín, Colombia \\ Email: carlosarturosj@gmail.com
}

Recibido: 6 de junio de 2019 | Aprobado: 18 de septiembre de 2019

https://doi.org/10.17533/udea.ef.n61a04

Resumen: El artículo examina el proceso de configuración del concepto de destrucción en las lecciones tempranas de Martin Heidegger entre 1919 y 1920. En este texto se sostiene que a través del establecimiento del marco fenomenológico del que este concepto surge se puede llegar a comprender mejor el proyecto filosófico de Heidegger y, sobre todo, la relevancia o vigencia que este proyecto tiene hoy. Primero, se establecen algunas de las fuentes de este concepto, particularmente en lo que se refiere al concepto de Abbau en Husserl. Luego, se aborda la articulación en las lecciones de 1919 y 1920 y, finalmente, se plantean algunas consideraciones críticas.

Palabras clave: fenomenología, Heidegger, Husserl, significatividad, destrucción, Abbau

* El artículo pertenece al Grupo de Investigación La hermenéutica en la discusión filosófica contemporánea, reconocido por COLCIENCIAS en la categoría A, código COL0064191 (avalado por la Universidad Nacional de Colombia, la Universidad de Antioquia y la Universidad de los Andes). Una versión preliminar fue presentada como ponencia en el Simposio "El pensamiento de Heidegger de cara al siglo XXl" organizado en el marco del VII Congreso Colombiano de Filosofía con el auspicio de la Sociedad Iberoamericana de Estudios Heideggerianos (SIEH), que se llevó a cabo en la ciudad de Bucaramanga del 15 al 18 de agosto de 2018.

Cómo citar este artículo:

Bedoya Rodas, C. A. (2020). Consideraciones sobre el concepto de destrucción fenomenológica en Martin Heidegger. A cien años de las lecciones de 1919-1920. Estudios de filosofía, 61, 35-54. https://doi.org/10.17533/udea.ef.n61a04

\section{OPEN \& ACCESS}




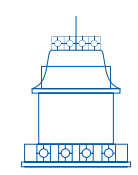

ARTÍCULOS

DE INVESTIGACIÓN

\title{
Remarks on the concept of phenomenological destruction in Martin Heidegger. One hundred years after the lectures of 1919-1920
}

\begin{abstract}
This article examines the genesis of the concept of destruction in the early lectures of Martin Heidegger between 1919 and 1920. The article claims that, by analyzing the phenomenological framework from which that concept arises, it is possible to reach a better understanding of Heidegger's philosophical work and its relevance today. The article traces some of the sources of this concept, in particular with reference to Husserl's concept of Abbau. Subsequently, the paper studies the articulation in the lectures of 1919 and 1920, and finally it puts forward some critical remarks.
\end{abstract}

Keywords: phenomenology, Heidegger, Husserl, meaningfulness, destruction, Abbau

\section{Carlos Arturo Bedoya Rodas}

Magíster en Filosofía de la Universidad de Antioquia. Licenciado en Filosofía de la Pontificia Universidad Javeriana. Docente de cátedra de la Facultad de Filosofía y del Centro de Humanidades de la Universidad Pontificia Bolivariana y del Instituto de Filosofía de la Universidad de Antioquia. Forma parte del grupo interinstitucional de investigación "La hermenéutica en la discusión filosófica contemporánea" avalado por la Universidad Nacional de Colombia, la Universidad de Antioquia y la Universidad de los Andes. Ha sido editor del volumen VIII del Anuario Colombiano de Fenomenología. Entre sus publicaciones más recientes: "De la ontología del mismo a la metafísica de lo otro: de Heidegger a Levinas" (En: Alteridad y formación, Editorial Bonaventuriana, 2017); "Vida e historia: La presencia de la Segunda intempestiva de Nietzsche en la hermenéutica temprana de Heidegger" (Revista Escritos, 2018); "Ontología y hermenéutica de la facticidad" (Hermenéutica del Long détour. Interpretaciones de la cultura, Editorial Universidad Pontificia Bolivariana, 2019). 


\section{Introducción}

Al hilo de la articulación del concepto de destrucción que se plasma en los textos de las lecciones impartidas por Martin Heidegger entre 1919-1920, el artículo quiere aproximarse a un modo de concebir la actividad filosófica que se pone en ejecución en estas lecciones y que ha llegado a ser considerado como una de las fuentes del pensamiento del siglo XX. A cien años del inicio de la actividad docente de Heidegger, sus lecciones tempranas, impartidas en Friburgo a comienzos de la década de 1920 y cuyos textos fueron publicados a finales de la década de 1980 y comienzos de la década de 1990, continúan suscitando interés para algunos por su carácter problemático y polémico, para otros, en cambio, representan un significativo y novedoso testimonio de la apropiación de la radicalidad de la actitud filosófica.

Pese al carácter fragmentario o esquemático de los textos de estas lecciones o, en ocasiones, al escaso desarrollo explícito de ciertos términos o nociones mencionadas, las siguientes páginas quieren alcanzar una aproximación al proceso de configuración del concepto de destrucción, el cual, desempeña un lugar central en estas lecciones. Con lo anterior se pretende tanto una comprensión del alcance y de los límites del modo como el filósofo alemán concebía durante esta etapa temprana el ejercicio filosófico, como de la vigencia que tal modo pueda seguir teniendo hoy.

Aunque el concepto de destrucción logra una mayor y definitiva articulación entre los años de 1922 con el Informe Natorp y en el curso de 1923 sobre la Hermenéutica de la facticidad, este artículo se centra en los textos de las lecciones de los años 1919 y 1920, y esto porque, en primer lugar, la presente interpretación de los textos de las lecciones quiere reivindicar el lugar fundamental que desempeñan estos primeros años de docencia para la comprensión del proyecto filosófico inicial. Gran parte de la ya clásica interpretación especializada sobre la hermenéutica temprana de Heidegger se ha concentrado preponderantemente en los textos y lecciones finales de la primera etapa de Friburgo, y ha hecho de estos el suelo explicativo para la comprensión del denominado pensamiento temprano de Heidegger (Gutiérrez, 1998; Kisiel, 2010; Rodríguez, 1997; Segura, 2002; Van Buren, 1994a). En segundo lugar, estos primeros cursos permiten establecer de manera más evidente el marco fenomenológico de gestación del procedimiento metódico de la destrucción.

En este artículo se pretende atender a la ausencia de referencias fenomenológicas más explícitas en el abordaje de este concepto por parte de la bibliografía especializada. Así, concretamente, en la aproximación a estas lecciones se intenta establecer el modo como en la articulación de este concepto se lleva a cabo una de las maneras en que tempranamente Heidegger se apropia de la fenomenología husserliana. La explicitación del marco fenomenológico en el que se elabora esta operación destructiva proporciona una comprensión más adecuada y, sobre todo, permite establecer de manera más evidente sus tensiones, alcances y límites, así como los del proyecto filosófico temprano del pensador de la Selva Negra en la medida en que se plasma en los textos de sus lecciones. 


\section{La destrucción en el pensamiento de Heidegger}

Es conocido el alcance que este concepto ha tenido a partir de la publicación en 1927 de Ser y tiempo para el modo en que se entiende el sentido del trabajo filosófico en el horizonte del pensamiento contemporáneo. Lo anterior se evidencia, por ejemplo, en el uso que Jacques Derrida hace del término "Deconstrucción". Según el filósofo francés, al acuñar este concepto deseaba traducir y adaptar para su propósito los términos heideggerianos de Destruktion y de Abbau, los cuales hacían referencia a una operación relativa a la estructura o arquitectura tradicional de los conceptos fundadores de la ontología o de la metafísica occidental (Derrida, 1989, p. 86).

Al atender con cuidado a la génesis del concepto de destrucción en el pensamiento de Heidegger se encuentra que este no siempre ha asumido la forma en la que se presenta en el muy conocido proyecto de Ser y tiempo, en el cual, al hilo de la pregunta por el ser, se articula como una confrontación con la historia de la ontología (Heidegger, 2003, p. 46). En los cursos de comienzos de la década de 1920 si bien la destrucción se encuentra referida a una forma de apropiación de la tradición filosófica y en esa medida presenta una cierta correspondencia con el carácter que este concepto asume en el proyecto de 1927, no se encuentra todavía suscrita en sentido estricto al que es el hilo conductor de este proyecto, es decir, al planteamiento de la pregunta por el sentido del ser. Por lo tanto, a diferencia de la manera como resulta articulada en el proyecto de una Ontología fundamental (1927), la destrucción en el joven Heidegger no se lleva a cabo tanto de modo primario como un trabajo destructivo de la historia de la ontología, vale decir, de algunas de las etapas decisivas de esta historia, cuanto como un ejercicio crítico que busca aclarar el ser de la vida fáctica. ${ }^{1}$

El concepto de destrucción es uno de los ejes constitutivos de todo el proyecto inicial de Martin Heidegger. Este concepto, que se gesta en el temprano proyecto de la fenomenología hermenéutica de la facticidad como una de sus líneas de cohesión y que designa aquello en lo que consiste la tarea propia de la investigación filosófica, hace manifiesta la preocupación constante por la apropiación del sentido del filosofar para el joven filósofo. Una confirmación de que en este concepto se juega un sentido renovado de la filosofía que puede ser relevante para la comprensión de la filosofía contemporánea y de los alcances y límites que esta forma de filosofar asume en el siglo XXI, viene señalada por el propio testimonio de Gadamer:

1 Una consideración más amplia acerca de la diferencia entre el concepto de ontología que se hace presente en el proyecto inicial de Heidegger y el modo como este opera en Ser y tiempo ha sido planteada por Hans-Helmuth Gander, quien sostiene que esta inicial ontología se orienta en la dirección de una hermenéutica del sí mismo (Gander, 2017, p. 228). En efecto, según este especialista, de manera distinta que la Ontología fundamental de 1927, la pregunta por el ser en el joven Heidegger debe entenderse como una pregunta por el ser de carácter antropológico o también hermenéutico-existencial (Gander, 2008, pp. 145-146). 
¿Qué era lo que nos atraía a mí y a otros hacia Heidegger? Entonces no lo supe decir, obviamente. Hoy lo veo así: Heidegger hacía asistir a la génesis de los esquemas de la tradición filosófica, presentándolos como respuestas a unas preguntas reales. El descubrimiento de la historia de su motivación daba a estas preguntas un carácter de ineludibilidad. Las cuestiones comprendidas no son meros objetos de conocimiento. Se convierten en verdaderas preguntas [...] Sólo cuando aprendí en Heidegger a conducir el pensamiento histórico a la recuperación de los planteamientos de la tradición, las viejas cuestiones resultaban tan comprensibles y vivas que se convertían en verdaderas preguntas. Lo que estoy describiendo es la experiencia hermenéutica fundamental, como la Ilamaría hoy (Gadamer, 1998, pp. 379-380).

\section{Las fuentes de la destrucción}

El término "destrucción" (Destruktion), que aparece por primera vez en las lecciones que Heidegger imparte entre 1919 y 1920, se corresponde con una germanización de la palabra latina destructio (Crowe, 2006, p. 45). Aunque Heidegger parece desconocer la prehistoria de esta palabra, para Dermont Moran es importante anotar que emerge como término filosófico de una inexacta traducción de un libro de Algazel, Destructio philosophorum, filósofo persa del siglo XI (Moran, 1994). En esta obra se critica la filosofía aristotélica por su arrogancia frente a la verdad revelada. Sin embargo, el filósofo Averroes, en una defensa de la naturaleza de la filosofía, refuta a Algazel a través de otra obra que fue traducida al latín como Destructio destructionis. De este modo, Moran señala que el término destrucción aparece en un contexto filosófico de debate entre la fe y la razón (Moran, 1994, pp. 175-196). En correspondencia con este marco, John Van Buren (1994a; 1994b) desde hace algunas décadas ha mostrado que los orígenes del uso de este término en Heidegger tienen relación con la destructio luterana, más particularmente, con el ataque de Lutero a Aristóteles y a la Escolástica.

Según Van Buren, el uso temprano por parte de Heidegger del término Destruktion no surge solamente de la noción kantiana de "crítica" sino que, sobre todo, resulta inspirada por los escritos de Lutero en la conocida Disputación de Heidelberg de 1518. En este sentido, el primer uso del término en el curso del semestre de invierno de 1919-1920 se encuentra realmente referido a la destructio luterana de la filosofía aristotélica. Del mismo modo, tanto las lecciones sobre la fenomenología de la vida religiosa de 19201921, como los cursos sobre Aristóteles a partir de 1921-1922 se inspiran en el modelo de destrucción luterana (Van Buren, 1994a, p. 167; 1994b, p. 172). Lutero pretende llevar a cabo un retorno a la experiencia original del cristianismo frente a la penetración que ha tenido este por la metafísica griega. De ahí que, en una apropiación de la teología paulina, pretenda desmantelar o destruir (destruere) los constructos especulativos 
para retornar a la experiencia kairológica y escatológica del cristianismo primitivo. A partir de este modelo, Van Buren parece encontrar la inspiración de la explosiva fuerza del concepto heideggeriano de destrucción del ser como presencia en la historia de la metafísica y de la tesis del "olvido del ser". Con esto, el especialista señala la relevancia de los orígenes teológicos y antimetafísicos en el pensamiento del filósofo alemán (Van Buren, 1994a, p. 202). ${ }^{2}$

Un tratamiento más sistemático y detallado del concepto de destructio en la obra de Lutero y una comparación con la destrucción en Heidegger la ha llevado a cabo de modo más reciente Benjamin D. Crowe. Este especialista se propone demostrar que una comprensión adecuada de la destrucción pasa por establecer las raíces luteranas del concepto, de este modo se enfoca en los textos y cursos de Lutero entre 1515 y 1518 y encuentra que los orígenes de la destrucción en Heidegger residen en una apropiación de la "teología de la cruz" elaborada por Lutero. Así, de un modo más detallado y sustentado que Van Buren, Crowe encuentra que en la Destruktion filosófica de Heidegger sobrevive algo del sentido original de la destructio luterana. De modo particular, el planteamiento de Crowe consiste en que, como en Lutero, la destrucción en Heidegger se ve motivada por una lucha por llevar a cabo un modo de vida auténtico (Crowe, 2006, pp. 44-66, 231-236). Con ello, este autor pretende hacer justicia al sentido de un concepto que se ha dado por obvio para los estudiosos. Efectivamente, Crowe pretende mostrar que a través del establecimiento de la influencia de Lutero sobre Heidegger llega a ser evidente la motivación práctica de la ejecución de la destrucción, esto es, que con ella se intenta responder a la cuestión acerca del papel y la responsabilidad de la filosofía en su propio tiempo (Crowe, 2006, pp. 2-3, 162-203).

Por su parte, Charles R. Bambach reconoce que el uso que hace Heidegger del concepto de destrucción se corresponde con una interpretación del ataque de Lutero a la teología escolástica que el joven filósofo alemán dirige, en cambio, hacia la filosofía contemporánea. Efectivamente, Bambach señala que la recuperación de la destrucción luterana por parte de Heidegger se transforma en una radical decisión entre la historia de la filosofía como una mera historia cultural e intelectual o la ejecución de la filosofía dentro de la facticidad de la vida contemporánea (Bambach, 1995, pp. 211ss). Entre tanto, el abordaje que Jeffrey Andrew Barash realiza de la destrucción heideggeriana se plantea desde el marco de problemas de la tradición historicista. De este modo, el especialista encuentra que el surgimiento de este concepto se produce en el intento de Heidegger por adaptar la filosofía a una función cultural y, sobre todo, por llevar a cabo una valoración crítica de la connotación específicamente filosófica que el término "cultura" había adquirido durante el siglo XIX (Barash, 1994, pp. 112-113).

2 Según Steven Crowell (2001, pp. 7-10), la interpretación de Van Buren parece quizá ser excesiva en cuanto que asevera una enorme influencia de Lutero en el joven Heidegger y esto porque, determinado por una perspectiva derridiana, Van Buren defiende una interpretación del joven Heidegger como "mística" y "antifilosófica". 
Pese a que los anteriores estudios parecen coincidir en algunas líneas generales respecto a las fuentes de la destrucción en el pensamiento inicial de Heidegger, resulta llamativo que no aborden explícitamente en sus acercamientos las referencias fenomenológicas del término. Tanto el abundante material textual del que hoy se dispone como el mismo sentido que expresa este central concepto permiten asegurar con certeza que la fenomenología husserliana y su apropiación por parte de Heidegger se constituyen en uno de los referentes decisivos para la elaboración y articulación de tal procedimiento metódico. Una muestra de ello se encuentra en el concepto del Abbau que hace presencia en los desarrollos fenomenológicos de Edmund Husserl ya desde las lecciones de 1919 y comienzos de 1920.

\section{El Abbau husserliano}

Frente a la opinión según la cual el uso del término Abbau por parte de Husserl aparecía de manera más tardía en la década de 1930, por ejemplo, en una obra como Experiencia y juicio (Husserl, 1980), ${ }^{3}$ los especialistas están hoy de acuerdo en que el término es introducido propiamente desde la década de 1920 (Evans, 1990, pp. 14-25; Gander, 2010, pp. 17-18; Staiti, 2010, pp. 130-132). En uno de sus usos más destacados, el término aparece como un procedimiento metodológico para el análisis de la génesis de la experiencia del mundo; dicho de manera más concreta, este procedimiento se dirige a poner de relieve o desmontar las distintas capas que constituyen el mundo de la experiencia. El uso que el Abbau tiene a partir de 1920 se circunscribe al contexto de una indagación sobre la demarcación de las regiones de la naturaleza (Natur) y del espíritu (Geist), como dan cuenta las lecciones alrededor de 1919.4 Este método que se pone en juego como un regreso a la experiencia preteorética o mundo de la vida, en tanto que la esfera en que las distinciones regionales se constituyen originariamente (Staiti, 2014, p. 141).

Según el importante Excurso de las lecciones de ética de 1920 aparecido en Hua XXXVII, este regreso o desmontaje se dirige hacia el estrato o capa más básica del mundo experimentado, es decir, a un estrato o esfera pasiva que soporta sin embargo todas las

3 En la amplia aproximación que realiza Dermont Moran (1994) a las fuentes de la destrucción en Heidegger este especialista señala que el Abbau aparece tan solo hasta el tardío texto de Husserl publicado en 1938. Del mismo modo, establece la relación directa entre este concepto y el de reducción fenomenológica. En este punto, la interpretación de Moran sigue al texto de Rodolphe Gasché (1986, pp. 109ss), quien, siguiendo a su vez la interpretación de David Carr (1974), indicaba la necesidad de volver a trazar el concepto derridiano de Deconstrucción desde el Abbau en la obra tardía de Husserl y la Destruktion en la obra temprana de Heidegger.

4 Como lo señala Ángel Xolocotzi (2016, p. 85), durante este año de 1919 las investigaciones de Husserl se difundieron así: Natur und Geist. Vorlesungen Sommersemester 1919, publicado como volumen IV de Husserliana Materialien en 2002. Conferencia "Natur und Geist", leída el 21 de febrero en la Sociedad de Ciencias Culturales de Friburgo, publicada en el volumen XXV de Husserliana en 1987. El manuscrito "Naturwissenschaftliche Psychologie, Geisteswissenschaften und Metaphysik" (1919) editado por Ullrich Melle y publicado en el libro Issues in Husserl's Ideas II en 1996. Die Lebenswelt. Auslegungen der vorgegebenen Welt und ihrer Konstitution, publicado como volumen XXXIX de Husserliana en 2008. 
demás capas, de las cuales a su vez surgen todas las determinaciones predicativas y valorativas. Esta capa básica es hacia la que se dirige el Abbau y que Husserl, en el famoso Excurso, denomina como "naturaleza pura" (Husserl, 2004, Hua XXXVIII, p. 296). Para alcanzar este estrato es necesario el desmonte de las capas cognitivas (Wissenschichten) y de los caracteres de valor y sentimiento (Wert und Gefühlscharakter) (Husserl, 2004, Hua XXXVII, p. 294). Las primeras capas se refieren a la esfera cognoscitiva, es decir, la del determinar y la del expresar, en la que el objeto aparece como el sustrato de posibles determinaciones predicativas (Husserl, 2004, Hua XXXVII, p. 287). La fase siguiente del desmontaje se dirige a los componentes emocionales o, en palabras de Husserl, a la "coloración emocional" (Gefühlsfärbung) (Husserl, 2004, Hua XXXVII, p. 294), desde la cual todo objeto es experimentado. El Abbau desmantela incluso esta capa permitiendo así la aparición de una esfera de experiencia pura. Esta esfera es obtenida a través de la exclusión de lo que el fundador de la fenomenología designa como "predicados de significación" (Prädikate der Bedeutung) (Husserl, 2002, Mat IV, p. 122). ${ }^{5}$

Por otra parte, como lo señaló Evans (1990, pp. 14-25) hace ya unas décadas y de modo más reciente Staiti (2010, p. 130), esta operación de desmonte desarrollada desde principio de la década de 1920 no se corresponde exactamente con la reducción fenomenológica. Como es conocido y dicho en términos generales, la reducción permite acceder a la esfera de la subjetividad trascendental, pero no significa que con ello la reducción ejecute por sí misma el desmontaje de los estratos cognitivos y emocionales (Staiti, 2010, p. 131). En efecto, el Abbau opera dentro del campo abierto por la reducción, pero no es idéntico a esta (Evans, 1990, p. 23). Mientras que la reducción deja que toda objetividad aparezca en su esencial correlación con la conciencia experienciada, el Abbau, según lo muestra Staiti, aprehende el más bajo o profundo de los estratos en que la esencial correlación es articulada (Staiti, 2010, p. 129). Al explicitar el concepto de desmontaje, Husserl repetidamente subraya que este es un método abstracto, esto es, que la capa más profunda de la naturaleza pura revelada a través de este método no es algo más real que la naturaleza dada en nuestra normal y no desmontada experiencia del mundo, sino que es más bien una dimensión de sentido (Staiti, 2010, p. 130). ${ }^{6}$

5 Como explica más claramente Xolocotzi: "Husserl señala la forma en la que nos relacionamos con las cosas y los cambios que se dan en los diversos modos de tratar con ellas. Así, los objetos pueden ser tema de nuevos actos que van desde la percepción hasta la apreciación de lo bello o la utilización práctica de algo. A los predicados añadidos en los diversos actos valorativos 0 prácticos Husserl los llama 'predicados de la significación'. [... ] diferenciar entre 'predicados reales' y 'predicados de significado'. Los últimos son a los que refiere a partir de las circunstancias en las que se presenta la cosa, mientras que los primeros buscan tematizar lo 'puro real'” (2016, pp. 91-92).

6 Este especialista señala que Heidegger y otros pensadores de la tradición hermenéutica tienden a confundir el método del desmontaje y la ejecución de la reducción fenomenológica. Según el autor, esta confusión conduce a la idea equivocada de que la realización de esta última podría causar una alteración de la experiencia vivida, excluyendo necesariamente sus características prácticas y estéticas, lo que luego sería inaccesible para una mirada reflexiva (Staiti, 2010, p. 131). 


\section{Mundo, significatividad y situación}

Desde su primer curso, en el denominado semestre de emergencia de 1919, y en el marco de la elaboración de una ciencia originaria (Urwissenschaft), Heidegger hace presente en los análisis de manera muy destacada el carácter de significatividad de la experiencia humana. Como lo muestra el filósofo en la conocida descripción de la vivencia del mundo circundante (Umwelterlebnis) (Heidegger, 2005a, p. 86) la significatividad (Bedeutsamkeit) se articula como el carácter que lleva todo contenido que se experimenta en la vida y por el cual todo se expresa de "algún modo". Este carácter es la manera siempre familiar en el que aparecen las cosas y esto debido a la profunda imbricación entre lo vivido y el yo que lo vive. Según Heidegger, la significatividad implica una resonancia y consonancia con el yo que no se encuentra bajo el esquema sujeto-objeto, sino que está antes de cualquier relación meramente cognoscitiva (Heidegger, 2005a, p. 91).

Lo anterior parece contradecir la conocida imagen en la que se considera que el mundo, conformado por objetos sin significación, es independiente de la vida. Según esta imagen, los objetos del mundo aparecen en principio carentes de sentido y, luego, a través de una operación cognoscitiva, aparecen para la vida como revestidos de significatividad (Heidegger, 2005b, pp. 47-51). De modo contrario, según lo muestran varios lugares de las lecciones al inicio de la década de 1920, la significatividad no es una esfera derivada sino precisamente la manera originaria en que aparece el mundo para la vida.

Por otra parte, como se señalaba, este carácter de significatividad también indica un modo familiar en el que aparece el mundo, vale decir, que lo que se experimenta no resulta del todo extraño y esto porque lo experimentado involucra o implica (Heidegger, 2013, pp. 166, 258). En este sentido, en lo que Heidegger viene a designar como la experiencia fáctica de la vida (faktische Lebenserfahrung), el mundo no aparece como algo distinto de la vida, como si fueran objetos independientes uno al lado del otro, sino que, más bien, aparece como el mundo de la vida (Lebenswelt) (Heidegger, 2013, pp. 65, 70, 72-83), como lo que se vive, se experimenta, se padece, de lo que se preocupa y, sobre todo, como un estar involucrado, un estar vinculado con algo.

Este estar vinculado que se expresa en la significatividad de la experiencia humana es lo que paulatinamente se intenta articular en el sentido del concepto de situación (Situation), el cual comienza a cobrar un lugar decisivo en el esfuerzo por llevar a la comprensión el fenómeno del vivir histórico, aunque no tenga un desarrollo suficientemente sistemático en los textos de las lecciones. Por situación no se entiende pues primariamente una multiplicidad de contenidos objetivos estáticos que se experimentan en un tiempo y espacio delimitados, sino, sobre todo, el modo como se tiene siempre experiencia del mundo (Heidegger, 2013, pp. 73, 175), es decir, una cierta unidad de la experiencia, pero no como un proceso (Vorgang) objetivado, vale decir, que pueda contemplarse u observarse simplemente, sino como un acontecimiento (Ereignis) 
que sucede en primera persona, que implica o involucra siempre a alguien (Heidegger, 1987, pp. 205-206). En este sentido, en la situación se expresa una implicación entre el mundo y el vivirse a sí mismo, el cual, en términos de Heidegger, es siempre situacional o histórico (Heidegger, 1987, p. 206; 2005b, pp. 120-121).

Con lo anterior no se indica que la vida sea un desconcierto caótico, ni un vago principio de fuerza, sino que, en cuanto situación, se manifiesta siempre como una figura concreta con sentido, lo que implica que se hace comprensible (Heidegger, 2013, pp. 157-159). De ahí que, para el Heidegger de estas lecciones, la intuición fenomenológica se plantee en términos de una intuición hermenéutica (Hermeneutische Intuition) o comprensiva que se esfuerza en captar la situación concreta, histórica, en la que se encuentra expresada la totalidad de la vida (2005a, p. 141). ${ }^{7}$

\section{La pérdida de significatividad}

A partir de 1919, las lecciones de Heidegger se esfuerzan por hacer ver la transformación radical que sufre el ámbito de inmediatez significativa de la vida desde la actitud científica. Efectivamente, como lo destaca el curso de 1919/20, Problemas fundamentales de la fenomenología, desde la ciencia, la experiencia mundana se muestra como despojada de la significatividad en la medida en que lo experimentado aparece en desconexión con la relación vital y dinámica en que originariamente se experimenta. De este modo, el mundo, como aquello de lo que se tiene experiencia, aparece entonces más bien como algo que se encuentra disponible (Heidegger, 2013, pp. 80, 218), a partir del cual se puede usar como un objeto y desde el que se conforma un campo temático, tanto para la ciencia como para la filosofía. En este sentido, el joven filósofo considera que, desde la conformación científica, la vida entra en una tendencia des-vivificadora (Ent-leben), en la medida en que se pierde el vínculo entre lo que se experimenta y el experimentar mismo; ${ }^{8}$ dicho de otro modo, la relación de la vida con el mundo, que

7 La captación de las situaciones vitales en que se concreta la vida es la tarea que en algunas lecciones Heidegger parece asignar a la fenomenología. Efectivamente, esta captación es lo que se designa en términos fenomenológicos como "intuición", la cual no debe ser entendida como "intuición de objetos", pues su correlato no son cosas (Dinge), en cuanto determinables, fijas, estáticas, aisladas. De modo contrario, la "intuición auténtica" tiene como correlato situaciones vitales concretas, es decir, históricas. Por eso, la intuición de la que habla Heidegger tiene la forma de lo que en el curso de 1919/20 se designa de un modo más claro que en el semestre de 1919, como la "comprensión pura" (reinen Verstehens). Esta, a diferencia de la reducción fenomenológica de Husserl que, entendida en su sentido negativo, supone la abstención de cualquier toma de posición, exige, en cambio, "«participar» en la experiencia personal de la vida con mayor vitalidad e intimidad”. En otros términos, la comprensión que explicita la fenomenología tal y como el joven Heidegger se apropia de ella, supone "un acompañar intuitivo, un ir a lo largo del sentido". Esto significa que cuando la fenomenología se entrega 0 acompaña el fluir del vivir, o sea, cuando se entrega a determinada situación, lo que allí sale al encuentro es un determinado nexo de sentido que debe ser interpretado (Heidegger, 2013, pp. 148-149, 159, 239, 241, 245-246, 261, 269).

8 En algunas de estas lecciones se ofrecen descripciones muy concretas acerca de esta transformación de la experiencia fáctica, por ejemplo, el contraste entre la vivencia del astrónomo con la vivencia del coro de los viejos tebanos (Heidegger, 2005a, p. 90). Para ver otras descripciones: 2013, pp. 77-78, 88, 217, 219. 
se da primariamente de modo significativo, deja de ser dinámica, ejecutiva y personal para convertirse en una relación estable y objetiva (2005b, p. 51).

En el significativo curso del semestre de emergencia de 1919, La idea de la filosofía y el problema de la concepción del mundo, esta tendencia aparece tematizada en el marco de una elaboración de una ciencia preteorética. Heidegger encuentra que en las ciencias y, de modo particular, en la filosofía se lleva a cabo una absolutización de la actitud teórica (theoretische Einstellung), de la cual, precisamente, le viene a esta última el ideal de rigurosidad (Heidegger, 2005a, p. 133). En un tono expresivo, en estas lecciones (p. 108) se advierte el modo como desde tal actitud lo significativo es de-significado al punto de quedar reducido a una mera realidad, y el yo histórico, por su parte, es des-historizado hasta llegar a ser una cosidad:

En la cúspide de este proceso de teoretización sale a relucir el carácter totalmente vacío, meramente objetivo y formal de algo [...] Su sentido carece de cualquier referencia al contenido mundano, por muy radicalmente teoretizado que esté ese contenido. Este algo es lo absolutamente privado de mundo, extraño al mundo; es la esfera donde uno pierde el aliento y no puede vivir (Heidegger, 2005a, p. 136).

Sin embargo, lejos de cualquier reivindicación de tipo irracionalista, lo que se señala es la primacía que la actitud teórica ha tenido en cuanto se le considera el estrato básico y fundamental en el que se fundan las esferas restantes de la vida, cuando es más bien esta actitud la que tiene su origen en la vida (Heidegger, 2005a, p. 109). Con lo cual, como derivada, esta actitud remite a lo que en estas lecciones apenas se designa como algo "preteórico" (vortheoretisch), cuyo sentido descansa en la plenitud de la vida misma. De esta manera, mientras la actitud teorética es una posición que se toma y en la que se está solo en algunas ocasiones, la vida como totalidad es el suelo originario, el tejido permanente en lo que ya de hecho siempre se está.

En el curso de verano de 1920 sobre Fenomenología de la intuición y de la expresión, esta tendencia se designa como un desvanecimiento o palidecer (Verblassen) de la significatividad (Heidegger, 1993, pp. 37, 182), ${ }^{9}$ es decir, un desplazamiento en la manera en que la vida tiene experiencia del mundo. Como se ha dicho, con esta tendencia se expresa que aquello de lo que se tiene experiencia se desprende entonces de su relación de existencia (Existenzbezug) y se convierte en algo que se encuentra fácilmente disponible (Heidegger, 1993, pp. 37, 182-183). De acuerdo con Heidegger, las ciencias, las artes y la filosofía, en la medida en que son manifestaciones del movimiento de la vida, también se encuentran afectadas por este desvanecimiento. Esto se muestra de modo claro en las teorías científicas, las proposiciones, los conceptos y las explicaciones

9 También en el curso de 1919 sobre La esencia de la universidad y de los estudios académicos se hace referencia a la disolución del carácter situacional (Heidegger, 1987, p. 206). 
filosóficas que no son ejecutadas desde la experiencia originaria de la cual fueron tomadas, transmitidas y desarrolladas (Heidegger, 1993, pp. 37, 183).

Ahora bien, como ya más claramente se asegura en el curso de verano de 1920, esta tendencia de la ciencia, la cual es un nexo de expresión, es paradójicamente una figura misma de la vida, un modo como ella aparece, como ella se expresa. De acuerdo con esto, en su propio nexo de expresión la vida se desvanece. De ahí, pues, que el joven Heidegger sostenga que la vida fáctica "se da en una determinada deformación" (2013, pp. 248, 157). Frente a esto surge entonces la necesidad de que el ejercicio filosófico, como Heidegger lo entiende por estos años, se plantee en términos de un trabajo crítico que busque deshacer la objetivación de la vida misma en la que esta se deforma (Heidegger, 1993, p. 251).

\section{Destrucción como ejercicio crítico y genético}

Pese a las diversas denominaciones que recibe el ejercicio filosófico durante estas lecciones iniciales, ${ }^{10}$ resulta evidente que en estas la filosofía aparece como un ejercicio investigativo dirigido a la explicitación de la vida fáctica. De ahí que cobre tanta relevancia la preocupación por establecer un acceso adecuado a este fenómeno. Efectivamente, si la indagación filosófica tiene como pretensión la explicitación categorial del fenómeno del vivir entonces tiene que asegurarse de aprehender el modo como este se manifiesta, lo que implica mantenerse en la inmediatez significativa en la que se da. Para el Heidegger de estas lecciones, el acceso adecuado al ámbito de inmediatez y vitalidad solo puede ser ofrecido en la actitud propiamente fenomenológica, ya que es solo esta la que en su mantenerse fiel a lo que se da y en el modo en que se da, ${ }^{11}$ puede mostrar el fenómeno del vivir propiamente. ${ }^{12}$ Sin embargo, debido a esta deformación a que tiende la vida, la tarea

10 En el denominado semestre de emergencia de 1919, Heidegger se refiere a una ciencia originaria de la vida; 0, en el semestre siguiente, a una ciencia del origen; posteriormente, en el Informe Natorp de 1922, aparece la designación de Hermenéutica fenomenológica de la facticidad y, finalmente, en su último curso en Friburgo en el semestre de verano de 1923, el proyecto recibe el nombre con el que va a ser ampliamente conocido, a saber, el de hermenéutica de la facticidad.

11 Y esto en consonancia con el principio de todos los principios de la fenomenología husserliana, que el mismo Heidegger cita en el curso de 1919: "que todo lo que se nos brinda originariamente [...] en la intuición, hay que tomarlo simplemente como se da, pero también sólo dentro de los límites en que se da” (Husserl, 1995, p. 58; Heidegger, 2005a, p. 132).

12 Esta actitud fenomenológica no es entendida desde la posición teórico-objetivante que el joven Heidegger encuentra en la reflexión, y que para Husserl es parte constitutiva del método fenomenológico. Como se explicita a partir del conocido $§ 77$ de Ideas I, el nuevo campo que se abre con la epojé "se mueve íntegramente en actos de la reflexión" (Husserl, 1995, p. 172). Husserl otorga a la reflexión un papel fundamental dentro de la fenomenología, en tanto que método de investigación propio de esta. En efecto, la reflexión, que se constituye en el método de conciencia para la conciencia general, tiene el carácter de una modificación de la conciencia, en tanto que surge esencialmente de cambios de actitud a partir de los cuales una vivencia no reflejada, experimenta una cierta transformación, en el modo de la conciencia reflejada. De esta manera para el filósofo de Moravia, la reflexión constituye la tarea de la fenomenología y por lo cual esta logra ser una ciencia reflexiva. Tal ciencia consiste justamente en la indagación de modo sistemático de estos conjuntos de modificaciones de las vivencias (Husserl, 1995, pp. 172-176). 
fenomenológica debe proceder como una "crítica puramente deformativa" o, también, como una "destrucción crítica" (Heidegger, 2013, pp. 157, 256, 262).

En las lecciones que llevan por título Fenomenología y filosofía trascendental de los valores, del semestre de verano de 1919, se hace referencia a una "crítica fenomenológica". En estas se lleva a cabo una revisión de la filosofía trascendental de los valores en cuanto que, junto con la escuela de Marburgo, era la más importante corriente filosófica de aquel momento y, sobre todo, en cuanto que, según Heidegger y en continuidad con la crítica iniciada el semestre anterior, el efecto histórico de la filosofía de los valores produjo una prevalencia de lo axiológico y lo teórico en todas las esferas de la vida. De este modo, el curso no pretende llevar a cabo una simple yuxtaposición entre la fenomenología y la filosofía trascendental de los valores sino más bien una crítica fenomenológica de esta corriente (Heidegger, 1987, p. 127). Sin embargo, por esta crítica no se entiende "una demostración de contradicciones, de absurdos, incoherencias y falacias [...] sino que la proposición a criticar es comprendida desde donde proviene su sentido, la crítica es una escucha positiva de las motivaciones auténticas" (p. 126).

De este modo, la crítica no consiste en una operación negativa ${ }^{13}$ en la que se refuten posiciones sino en un llevar los problemas hacia lo que el Heidegger de esta época encuentra como el lugar genuino de la fenomenología, esto es, "la vida en y para si" (Heidegger, 1987, p. 121). Dicho de otro modo, la crítica fenomenológica, de un modo semejante al Abbau husserliano, consiste en una operación genética que se dirige a un ámbito no derivado, que, para el caso del Heidegger de estos años, se entiende como el ámbito o esfera vital.

Ahora bien, el uso de este término, crítica fenomenológica, parece pues anteceder a la aparición del término destrucción (Destruktion) en el semestre de invierno 1919/20. Sin embargo, en estas lecciones tal término solo se emplea en contadas ocasiones (Heidegger, 2013, pp. 139,154, 245, 248, 256, 262), ${ }^{14}$ siendo, más bien, en el curso de verano de 1920 en donde tal concepto recibe un desarrollo más explícito y en el que, por primera vez, se equipara con el uso del término Abbau. Efectivamente, la destrucción es presentada como un "desmontaje" (Abbau) (Heidegger, 1993, pp. 35, 181), lo cual excluye, en principio, un sentido de "rompimiento" (Zerschlagen) crítico, o de un "demolimiento" (Zertrümmern).

Este procedimiento responde a lo que antes se ha caracterizado como el desvanecimiento o el palidecer de la significatividad, el cual ha sido visto como una tendencia de la vida en su facticidad que afecta la propia conceptualidad filosófica, en la medida en que esta es también una expresión de la vida. Lo anterior supone que las proposiciones y conceptos

13 En el semestre siguiente Heidegger aborda el carácter negativo de la destrucción. Un completo abordaje de este carácter y de su relación con la dialéctica puede encontrarse en De Lara (2009).

14 Un contexto y tratamiento más amplio sobre la destrucción puede encontrarse en Bedoya (2017). 
filosóficos no disponen ya de la fuente de sentido desde la que fueron tomados, transmitidos y desarrollados; lo que en términos de este curso se plantea como la relación originaria de existencia (Existenzbezug) (Heidegger, 1993, pp. 37, 183).

La destrucción se plantea como desmontaje en cuanto que, en consonancia con el radical "retroceso «a las cosas mismas»" (Rückgang zu »den Sachen selbst «) (Heidegger, 1993, p. 30) de la fenomenología, pretende un regreso o retroceso a las fuentes originarias de sentido a partir de las cuales se han formado los conceptos que constituyen las perspectivas interpretativas que guían la investigación filosófica. Esto significa que la destrucción crítica fenomenológica, como otra designación que se le da en estas lecciones, debe llevar los conceptos a lo que el autor refiere en algunas lecciones bajo el nombre de una experiencia fundamental (Heidegger, 1993, pp. 35, 180, 187; 2013, p. 109; 2000, p. 36).

De modo concreto, como es expuesto en este curso de verano de 1920, el ejercicio destructivo se encuentra inicialmente con conceptos y significados que se han transmitido por la tradición (Heidegger, 1993, pp. 51, 59, 80-81) 15 $^{15}$ que, por ende, se ven envueltos en una condición de ambigüedad. La efectuación de la destrucción no se dedica a una clarificación técnica de los significados de las palabras, tomándolos como objetos separados del campo de sentido que les dio origen (p. 179) ya que, tal como el joven Heidegger lo señala, el significado de un concepto no es un objeto que se determina teóricamente, sino que debe ser comprendido existencialmente (p. 187). Así pues, lo que el trabajo conceptual pretende es una comprensión vital de los significados (p. 179), es decir, una comprensión ejecutiva. ${ }^{16}$ Los significados no son concebidos, por lo tanto, como relaciones objetivamente determinadas que existen per se, sino que se encuentran remitidos a un contexto concreto o situación de sentido desde el que se ejecutan (p. 179).

La destrucción, entonces, aunque se lleve a cabo inicialmente como un trabajo de orden conceptual, no se encuentra al servicio de fines cognoscitivos (Heidegger, 1993, pp. 178, 185). El trabajo destructivo, en cambio, pertenece a la vida fáctica; se encuentra motivado existencialmente desde ella como un modo de respuesta a la tendencia objetivante-desvivificadora que afecta al vivir, tanto como a la explicitación filosófica. ${ }^{17}$

15 Un desarrollo más amplio sobre la tradición y el ejercicio destructivo en este semestre y, sobre todo, en los siguientes años, en los cuales la destrucción aparecerá ya propiamente configurada en su referencia a una confrontación con la tradición filosófica puede ser consultado en Bedoya, C.A \& Guardia, A. (2018).

16 A través del uso del término latino Diiudication, según Heidegger, el criterio para determinar el carácter originario del sentido ejecutivo consiste en que este se encuentre dirigido al mundo del sí-mismo y que entrañe siempre la exigencia de renovación (Erneuerung) (Heidegger, 1993, p. 75). Véase: Hogemann (1988). Alejandro Vigo, por su parte, encuentra en esta caracterización del sentido ejecutivo una relación con el actus exercitus, uno de los términos de la conocida distinción agustiniana (Vigo, 2008, p. 250). Con este término se indica un ir al discurso expresado para escuchar su motivación secreta y su trasfondo, pero de igual modo saber cómo aplicar lo que ha sido expresado a la propia situación concreta. De esta manera, solamente el esfuerzo del actus exercitus permite llegar al pleno sentido de las proposiciones. Lo anterior es lo que, de acuerdo con Grondin, se encuentra en el sentido que tiene la comprensión de los conceptos filosóficos para el joven Heidegger (Grondin, 1995, pp. 148-149).

17 Sobre el decisivo concepto de motivación en las tempranas lecciones y sus referencias fenomenológicas puede consultarse: Garcés Ferrer (2018). 
Según Heidegger, el resultado del ejercicio destructivo no consiste en el descubrimiento de lo que podría parecer, en términos del proyecto husserliano, como de una "esencia" o de una necesidad absoluta (p.185), sino más bien, en la recuperación de la experiencia fundamental de la vida fáctica. Esta experiencia no es un tipo de iluminación especial que sucede a algunos en ciertas ocasiones, sino que, en palabras del propio autor, "es posible en todo concreto vivir fáctico donde la preocupación [Bekümmerung] misma se retrotrae [zurückbringt] al vivir actual" (p. 174). Sin duda esto se corresponde con lo que en los cursos entre 1919 y 1920 se señala como la existencia siempre concreta y propia de cada uno (p. 173). Según lo expresan de distintas maneras estas lecciones iniciales de los años de 1919 y 1920, es desde esta experiencia desde donde surge toda indagación y conceptualidad filosófica (Heidegger, 1993, pp. 174, 179), y a la que, a su vez, permanece vinculada la destrucción. ${ }^{18}$

El concepto de destrucción tal y como aparece articulado en los textos de clases de estos años (1919-1920) permite ver una constante preocupación por el modo de apropiación del ejercicio filosófico. Con el carácter fragmentario o esquemático con el que se presentan y pese a la variedad de formulaciones en el que se articulan, se hace posible destacar uno de los ejes sobre los que se configura el pensamiento temprano de Heidegger, esto es, el interés por una forma de comprender y, con ello, de poner en ejecución la actitud de radicalidad abierta por la fenomenología en su "regreso a las cosas mismas". Esto es precisamente lo que se permite ver en una aproximación al proceso como se configura el concepto de destrucción.

El reconocimiento del marco fenomenológico al que responde el sentido de este concepto muestra los horizontes y, con ello, las tensiones desde las que se configura esta operación crucial del proyecto filosófico del pensador alemán. Así, se hace más evidente que el ejercicio destructivo parece corresponderse al modo husserliano de un procedimiento genético que se dirige a un estrato no derivado. No obstante, mientras en Husserl se plantea como un ámbito de "pureza", su joven discípulo, en cambio, lo encuentra como "vital e histórico". Parece que las diferencias pueden encontrarse a partir de lo que en cada uno se plantea como "lo significativo", esto es, el sentido. La destrucción en los tempranos desarrollos del pensamiento de Heidegger puede ser considerada como un retroceso que, de modo inverso al de Husserl en las lecciones de 1919 y 1920, se plantea como una recuperación de lo significativo, en el marco de una preocupación por el carácter existencial o ejecutivo que corresponda al modo radical en que se constituye la actitud fenomenológica. Por último, el reconocimiento del marco en el que se configura la destrucción no solo permite establecer las diferencias en los modos de apropiación de actitud fenomenológica sino, y de manera significativa, advertir

18 "De acuerdo con su sentido, una destrucción tal permanece vinculada a la preocupación de sí mismo concretamente propia y plenamente histórica" (Heidegger, 2000, p. 34). 
que la actitud crítica de la fenomenología en su abordaje de la experiencia humana implica un ejercicio genético dirigido a la apropiación de los ámbitos de sentido y que se presuponen como ya establecidos y consolidados.

\section{Algunas consideraciones críticas}

Lo expuesto hasta aquí parece confirmar lo que desde hace algunos años Francisco De Lara ha destacado en el marco de una discusión crítica acerca del trabajo filosófico según el joven Heidegger (De Lara, 2014, 2016). De acuerdo con este especialista, particularmente en lo esbozado entre los cursos de 1919 y 1920 ( La idea de la filosofía y el problema de la cosmovisión, Problemas fundamentales de la fenomenología), la concepción filosófica que sostiene Heidegger "parece acentuar en exceso los elementos motivacionales-existentivos", lo cual "implica finalmente socavar la posibilidad de la filosofía como una tematización científica, esto es, que se pliega últimamente al asunto del que trata" (De Lara, 2014, p. 72). Y esto porque el mismo Heidegger parece poner en marcha una idea de fenomenología como "ciencia originaria" (Urwissenschaft) o como investigación radicalmente primera a la que no le corresponde dar nada por supuesto. En este sentido, lo esbozado ya en el curso de 1919 advertía un proyecto filosófico que estaba guiado por una idea formal, es decir, por la idea de una investigación libre de presupuestos (De Lara, 2014, p. 68).

Sin embargo, como señala De Lara, el proyecto filosófico del joven Heidegger pasa a ser un programa concreto de filosofía, que ya ha decidido de antemano su objeto, su motivación y su posible rendimiento (De Lara, 2014, p. 69). De hecho, como lo evidencia más claramente el curso de 1919/20, Problemas fundamentales de la fenomenología, Heidegger se guía por una idea particular de lo que debe ser el ejercicio filosófico, cuya motivación principal reside en la de responder radicalmente "a la pregunta existencial que cada cual es para sí mismo" (De Lara, 2014, p. 71). Desde esta inquietud originaria se articularía la motivación del ejercicio filosófico para el joven Heidegger. Según De Lara, y como en estas páginas se ha hecho evidente, la destrucción, como aspecto del método fenomenológico, no escapa a esta motivación.

De acuerdo con un cuidadoso análisis textual, la descripción que realiza De Lara acerca de la idea de filosofía que está en juego en la temprana obra de Heidegger, de manera particular en lo que respecta a la tensión entre una concepción como ciencia originaria (Urwissenschaft) o como ciencia del origen (Ursprungswissenschaft), resulta evidente. Asimismo, la discusión crítica que con esto se plantea resulta de una gran relevancia, sobre todo, si se trata de preguntarnos, entre otras cuestiones, por los alcances y límites del pensamiento de Heidegger de cara a los retos de la filosofía en el siglo XXI. 
Cabe pues preguntarse si, pese a los límites que se evidencian en esta concepción temprana de filosofía, un aspecto metodológico tan central como el de la destrucción -de cuya génesis las páginas anteriores han intentado realizar una aproximación- puede hoy seguir teniendo relevancia como un modo de llevar a cabo el trabajo filosófico. De acuerdo con esto, es preciso plantearse si la destrucción resulta lastrada definitivamente por los presupuestos de orden existentivo que la motivan, como ha hecho ver De Lara. 0 si, como bien lo ha indicado Courtine, la exigencia fenomenológica de captación cada vez renovada de algo dado, que parece dar forma al ejercicio destructivo en algunos lugares de las lecciones tempranas (Courtine, 2011, p. 23), puede continuar teniendo un alcance significativo para el modo de hacer filosofía hoy. Precisamente, en relación con esto, De Lara muestra que el sentido que tiene para el Heidegger de esta época la experiencia filosófica radica en última instancia en el "afán de problematizar, en no dar nada por hecho, en no asumir nada como válido ni aceptar lo heredado sin someterlo a una apropiación interrogativa" (De Lara, 2014, p. 71). Ahora bien, es justamente este el sentido al que responde el ejercicio destructivo, de ahí que más allá de considerarlo simplemente como un posible reflejo de una concepción individualista y elitista de la filosofía a la que Heidegger parece adscribirse, según lo plantea De Lara, en este central aspecto metodológico de su pensamiento inicial puede encontrarse una invitación y un desafío muy vigente hacia el cultivo y ejecución de una actitud crítica y, con ello, responsable.

\section{Referencias}

Bambach, C. R. (1995). Heidegger, Dilthey, and the Crisis of Historicism. Ithaca, N.Y.: Cornell University Press.

Barash, J. A. (1994). Heidegger's Ontological 'Destruction' of Western Intellectual Traditions. In T. Kisiel \& J. Van Buren (Eds.), Reading Heidegger from the Start: Essays in his Earliest Thought (pp. 111-122). New York: SUNY.

Bedoya, C. A. (2017). Recuperar la vida. Hermenéutica y destrucción en el joven Heidegger (1919-1923). Medellín: Editorial UPB.

Bedoya, C. A. \& Guardia, A. (2018). Vida e historia: La presencia de la Segunda intempestiva de Nietzsche en la hermenéutica temprana de Heidegger. Escritos 26(57), 369-388. http://dx.doi.org/10.18566/escr.v26n57.a06

Carr, D. (1974). Phenomenology and the Problem of History. Evanston: Northwestern University Press. Courtine, J. F. (2011). Reducción, construcción, destrucción. De un diálogo entre tres: Natorp, Husserl, Heidegger. En A. Rocha (Ed.), Heidegger hoy. Estudios y perspectivas (pp. 21-41). Buenos Aires: Grama Ediciones.

Crowe, B. D. (2006). Heidegger's Religious Origins. Destruction and Authenticity. Bloomington, IN: Indiana University Press. 
Crowell, S. G. (2001). Husserl, Heidegger and the Space of Meaning. Paths toward Transcendental Phenomenology. Evanston: Northwestern University Press. https:// doi.org/10.2307/j.ctv47wc7b

De Lara, F. (2009). Fenomenología y dialéctica. La crítica de Heidegger a la dialéctica. En A. Rocha (Ed.), Martin Heidegger. La experiencia del camino (pp. 95-123). Barranquilla: Ediciones Uninorte.

De Lara, F. (2014). ¿Ciencia originaria o ciencia del origen? Una discusión de la filosofía de Heidegger en sus cursos de 1919 a 1921. Revista de Humanidades 30, 55-75.

De Lara, F. (2016). ¿Fenomenología de lo Concreto? Crítica Fenomenológico-Política del Programa Filosófico de Heidegger en 1919/20. Trans/Form/Ação, 39(1), 37-56. http://dx.doi. org/10.1590/s0101-31732016000100003

Derrida, J. (1989). Carta a un amigo japonés. Suplementos Anthropos, 13, 86-89.

Evans, I. C. (1990). Phenomenological Deconstruction: Husserl's Method of Abbau. Journal of the British Society for Phenomenology, 21(1), 14-25. http://dx.doi.org/10.1080/00071 773.1990 .11006874

Gadamer, H.-G. (1998). Autopresentación de Hans-Georg Gadamer (1977). En Verdad y método II (M. Olasagasti, Trad.). Salamanca: Sígueme.

Gander, H.-H. (2008). La fenomenología hermenéutica del vivir fáctico de Heidegger. En F. Duque (Ed.), Heidegger. Sendas que vienen. (F. de Lara, Trad.) (pp. 139-171). Madrid: Círculo de bellas Artes.

Gander, H-H. (Ed.). (2010). Husserl-Lexikon. Darmstadt: WBG.

Gander, H-H. (2017). Self Understanding and Lifeworld. Basic Traits of a Phenomenological Hermeneutics. (R. Drake \& J. Rayman, Trad.). Bloomington, IN: Indiana University Press. https://doi.org/10.2307/j.ctt2005w6h

Gasché, P. (1986). The Tain of the Mirror. Derrida and the Philosophy Reflection. Cambridge: Harvard University Press.

Garcés Ferrer, R. (2018). El concepto de motivación en la fenomenología hermenéutica del joven Heidegger. Anales del Seminario de Historia de la Filosofía, 35(2), 439-458. https://doi. org/10.5209/ASHF.59663

Grondin, J. (1995). Sources of Hermeneutics. Albany: SUNY.

Gutiérrez, C. B. (1998). La hermenéutica temprana de Heidegger. Ideas y valores, 47(107), 27-42.

Heidegger, M. (1987). Zur Bestimmung der Philosophie. Frankfurt am Main: Vittorio Klostermann Verlag. [GA 56/57].

Heidegger, M. (1993). Phänomenologie der Anschauung und des Ausdrucks. Theorie der philosophischen Begriffsbildung. Frankfurt am Main: Vittorio Klostermann. [GA 59].

Heidegger, M. (2000). Anotaciones a la Psicología de las visiones del mundo de Karl Jaspers. En Hitos (H. Cortés \& A. Leyte, Trads.). Madrid: Alianza. [GA 9]. 
Heidegger, M. (2003). Ser y tiempo. (J. E. Rivera, Trad.). Madrid: Trotta.

Heidegger, M. (2005a). La idea de la filosofía y el problema de la concepción del mundo. (J. Adrián Escudero, Trad.). Barcelona: Herder. [GA 56/57].

Heidegger, M. (2005b). Introducción a la fenomenología de la religión. (J. Uscatescu, Trad.). Madrid: Siruela. [GA 60].

Heidegger, M. (2013). Problemas fundamentales de la fenomenología (1919/1920). (F. de Lara, Trad.). Madrid: Alianza. [GA 58].

Hogemann, F. (1988). Vida y alienación. Sobre la fenomenología de Heidegger a principios de los años veinte. En F. Duque (Ed.), Los confines de la modernidad (pp. 147-169). Barcelona: Ediciones Juan Granica.

Husserl, E. (1980). Experiencia y juicio. Investigaciones acerca de la genealogía de la lógica (J, Reuter Trad.). México: UNAM.

Husserl, E. (1995). Ideas relativas a una fenomenología pura y una filosofía fenomenológica. (J. Gaos, Trad.). México: Fondo de Cultura Económica.

Husserl, E. (2002). Natur und Geist. Vorlesungen Sommersemester 1919. Dordrecht: Kluwer Academic Publishers [Mat IV]. https://doi.org/10.1007/978-94-010-0429-9

Husserl, E. (2004). Einleitung in die Ethik, Vorlesungen Sommersemester 1920/1924. Dordrecht: Springer. [Hua XXXVII].

Kisiel, T (2010). Hermeneutics of facticity. In B. W. Davis (Ed.). Martin Heidegger: Key Concepts (pp. 17-32). Durham: Acumen. https://doi.org/10.1017/UPO9781844654475.002

Moran, D. (1994). The Destruction of the Destruction: Heidegger's Versions of the History of Philosophy. In K. Harries \& C. Jamme (Eds.), Martin Heidegger: Politics, Art and Technology (pp. 175-196). New York: Homes and Meier.

Rodríguez, R. (1997). La transformación hermenéutica de la fenomenología. Una interpretación de la obra temprana de Heidegger. Madrid: Tecnos.

Segura Peraita, C. (2002). Hermenéutica de la vida humana. En torno al Informe Natorp de Martin Heidegger. Madrid: Trotta.

Staiti, A. (2010). Different Worlds and Tendency to Concordance: Towards a New Perspective on Husserl's Phenomenology of Culture. In The New Yearbook for Phenomenology and Phenomenological Philosophy, 10 (pp. 127-142). New York: Routledge. https://doi. org/10.1558/nyppp.v10i1.127

Staiti, A. (2014). Husserl's Transcendental Phenomenology. Nature, Spirit and Life. Cambridge: Cambridge University Press. https://doi.org/10.1017/CBO9781107588875

Van Buren, J. (1994a). The Young Heidegger. Rumor of the Hidden King. Bloomington, IN: Indiana University Press.

Van Buren, J. (1994b). Martin Heidegger, Martin Luther. In T. Kisiel \& J. Van Buren (Eds.), Reading Heidegger from the Start: Essays in his Earliest Thought (pp. 159-174). New York: SUNY. 
Vigo, A. (2008). Arqueología y Aleteiología y otros estudios heideggerianos. Buenos Aires: Biblos. Xolocotzi, Á. (2016). Mundo circundante y mundo de vida en 1919. Contexto de la confrontación entre Husserl y Heidegger. En Heidegger-Husserl. Studia Heideggeriana, 5 (pp. 75-100). Buenos Aires: Teseo-Sociedad Iberoamericana de Estudios Heideggerianos. 